\title{
Forward-Invariant Peeling in Chemical Dynamics: a Simple Case Study
}

\author{
A.N. Gorban* \\ Department of Mathematics, University of Leicester, Leicester, LE1 7RH, UK
}

\begin{abstract}
Forward-invariant peeling aims to produce forward-invariant subset from a given set in phase space. The structure of chemical kinetic equations allows us to describe the general operations of the forward-invariant peeling. For example, we study a simple reaction network with three components $A_{1}, A_{2}, A_{3}$ and reactions $A_{1} \rightarrow A_{2} \rightarrow A_{3} \rightarrow A_{1}, 2 A_{1} \rightleftharpoons 3 A_{2}$ (without any stoichiometric conservation law). We assume that kinetics obey the classical mass action law and reaction rate constants are positive intervals $0<k_{i} \min \leq k_{i} \leq k_{i} \max <\infty$. Kinetics of this system is described by a system of differential inclusions. We produce forward-invariant sets for these kinetic inclusions from the sets $\left\{c \mid c_{i} \geq 0, \sum c_{i} \geq \varepsilon\right\}$ by the forward-invariant peeling (for sufficiently small $\varepsilon>0$ ). In particular, this construction proves persistence of this kinetic system (a positive solution cannot approach the origin even asymptotically, as $t \rightarrow \infty$ ).
\end{abstract}

Keywords and phrases: chemical kinetics, Lyapunov function, persistence

Mathematics Subject Classification: 37C10, 34D20, 93D05

\section{Introduction}

\subsection{The problem}

Differential inclusions for chemical kinetics with a given reaction mechanism, unknown rate constants but known thermodynamic data were introduced in 1979 [3] (for further references, generalizations and examples see $[2,4,5,7,8,10]$; our works were mostly inspired by A.I.Volpert [9]). Solutions to these inclusions are forward-invariant sets of the kinetic equations.

In many important cases, the cones of the possible velocities in the concentration spaces are piecewise constant (for systems with a given reaction mechanism and the complex balance condition but unknown values of reaction rate constants, for example [7]). Two main approaches to evaluation of solutions to these kinetic inclusion have been proposed. If we start from an initial point and build the solutions stepwise by adding the cones of the possible velocities and intersection with the compartments where these cones are constant then the result is the minimal forward-invariant set which includes the initial point. The dual approach is the so-called forward-invariant peeling: we start from a domain in the concentration space and then delete some parts of this set (the 'peel' or the 'rind') to make it invariant [7].

\footnotetext{
${ }^{*}$ Corresponding author. E-mail: ag153@le.ac.uk
} 
The goal of this note is to demonstrate how the procedure of forward-invariant peeling works on a simple but not yet trivial example. For this purpose, we take a system from a recent paper [8]

$$
A_{1} \stackrel{k_{7}}{\rightarrow} A_{2} \stackrel{k_{2}}{\rightarrow} A_{3} \stackrel{k_{3}}{\rightarrow} A_{1}, \quad 2 A_{1} \underset{k_{-4}}{\stackrel{k_{4}}{\rightleftharpoons}} 3 A_{2}
$$

with the classical mass action law and interval constants $0<\min k_{i} \leq k_{i} \leq \max k_{i}<\infty$. Consider the kinetic equations with such interval constants and classical mass action law. We will demonstrate how to use peeling for solution of the persistence problem for this system: is it possible that the solution of the differential inclusion with these interval constants starting from a positive vector will go to zero when $t \rightarrow \infty$ ? This question for that system was considered recently as an unsolved problem [8]. The solution was demonstrated in recent preprint [7]. The goal of this note is rather modest in the light of the general theory of toric inclusions announced very recently [1] but I believe that the publication of the straightforward analysis of an example of forward-invariant peeling still makes sense.

\subsection{Mass action law}

The list of components is a finite set of symbols $A_{1}, \ldots, A_{n}$.

A reaction mechanism is a finite set of the stoichiometric equations of elementary reactions:

$$
\sum_{i} \alpha_{\rho i} A_{i} \rightarrow \sum_{i} \beta_{\rho i} A_{i}
$$

where $\rho=1, \ldots, m$ is the reaction number and the stoichiometric coefficients $\alpha_{\rho i}, \beta_{\rho i}$ are nonnegative numbers. Usually, these numbers are assumed to be integer but in some applications the construction should be more flexible and admit real nonnegative values. Let $\alpha_{\rho}, \beta_{\rho}$ be the vectors with coordinates $\alpha_{\rho i}, \beta_{\rho i}$ correspondingly.

A stoichiometric vector $\gamma_{\rho}$ of the reaction in Equation (1.2) is a $n$-dimensional vector $\gamma_{\rho}=\beta_{\rho}-\alpha_{\rho}$ with coordinates

$$
\gamma_{\rho i}=\beta_{\rho i}-\alpha_{\rho i}
$$

that is, "gain minus loss" in the $\rho$ th elementary reaction. We assume $\alpha_{\rho} \neq \beta_{\rho}$ to avoid trivial reactions with zero $\gamma_{\rho}$.

A non-negative intensive variable, the reaction rate $r_{\rho}$, corresponds to each elementary reaction.

The kinetic equations for a homogeneous system in the absence of external fluxes are

$$
\frac{\mathrm{d} N}{\mathrm{~d} t}=V \sum_{\rho} r_{\rho} \gamma_{\rho} .
$$

If the volume is not constant then the equations for concentrations include $\dot{V}$ and have different form (this is typical for combustion reactions, for example). In our case study we assume that the volume is constant and the equation for concentration can be used

$$
\frac{\mathrm{d} c}{\mathrm{~d} t}=\sum_{\rho} r_{\rho} \gamma_{\rho}
$$

Mass Action Law (MAL) gives a representation of the reaction rate as the functions of concentrations:

$$
r_{\rho}=k_{\rho} \prod_{i=1}^{n} c_{i}^{\alpha_{\rho i}}
$$

where $k_{\rho}$ are kinetic constants (they depend on the temperature but not on the concentrations).

For the intervals of kinetic constants, we work with differential inclusions

$$
\frac{\mathrm{d} c}{\mathrm{~d} t} \in \sum_{\rho} r_{\rho} \gamma_{\rho} ; \min k_{\rho} \leq k_{\rho} \leq \max k_{\rho}
$$




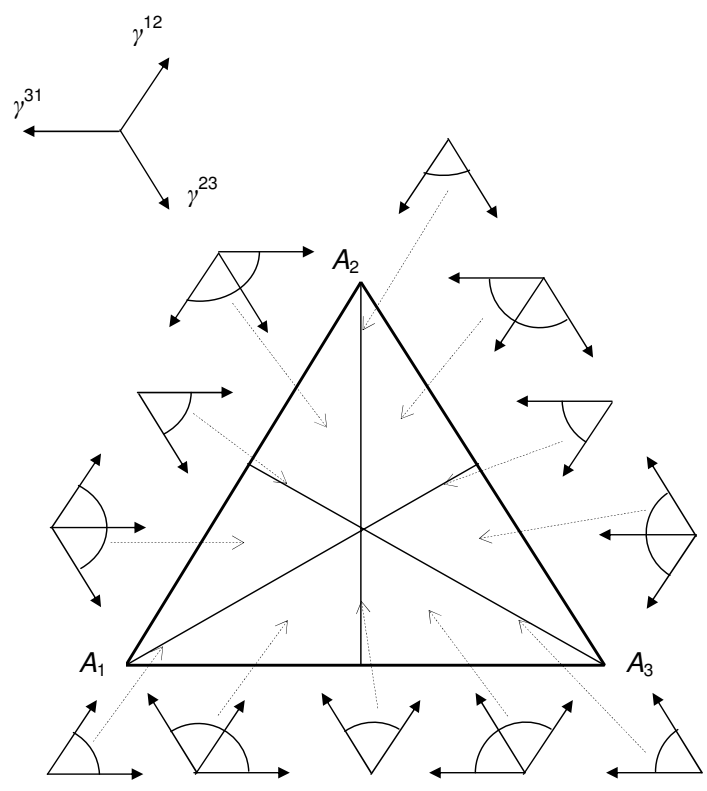

FiguRE 1. Kinetic inclusion for first order kinetics with three components and given equilibrium. The triangle of concentrations is split into compartments by the partial equilibria lines. The corresponding cones of possible vectors $\dot{c}$ (the angles) for given equilibrium are piecewise constant (without any assumption of detailed balance).

\section{A toy example}

\subsection{The system}

Let us consider a reaction mechanism (1.1) [8].

The stoichiometric vectors of the reactions are

$$
\gamma_{1}=\left(\begin{array}{c}
-1 \\
1 \\
0
\end{array}\right) ; \gamma_{2}=\left(\begin{array}{c}
0 \\
-1 \\
1
\end{array}\right) ; \gamma_{3}=\left(\begin{array}{c}
1 \\
0 \\
-1
\end{array}\right) ; \gamma_{4}=\gamma_{-4}=\left(\begin{array}{c}
-2 \\
3 \\
0
\end{array}\right)
$$

The reaction rates are: $r_{1}=k_{1} c_{1}, r_{2}=k_{2} c_{2}, r_{3}=k_{3} c_{3}, r_{4}=k_{4} c_{1}^{2}, r_{-4}=k_{-4} c_{2}^{3}$

The chemical kinetic inclusions for the given interval of constants are

$$
\frac{\mathrm{d} c}{\mathrm{~d} t} \in k_{1} c_{1}\left(\begin{array}{c}
-1 \\
1 \\
0
\end{array}\right)+k_{2} c_{2}\left(\begin{array}{c}
0 \\
-1 \\
1
\end{array}\right)+k_{3} c_{3}\left(\begin{array}{c}
1 \\
0 \\
-1
\end{array}\right)+\left(k_{4} c_{1}^{2}-k_{-4} c_{2}^{3}\right)\left(\begin{array}{c}
-2 \\
3 \\
0
\end{array}\right),
$$

where $k_{i} \in\left[\min k_{i}, \max k_{i}\right]$.

This system has no linear conservation law (like mass balance). In chemical kinetics such systems might appear as subsystems of larger systems. For example, we can assume that the nonlinear reaction $2 A_{1} \rightleftharpoons 3 A_{2}$ includes one more reagent, $B: 2 A_{1}+B \rightleftharpoons 3 A_{2}$ and the concentration of $B$ is kept constant.

The systems with total mass balance cannot approach zero from a positive initial state, this means, it is persistent. Without such a conservation law persistence depends on the reaction mechanism. Nevertheless, we will demonstrate that solution of inclusion 2.2 cannot tend to zero from positive initial conditions. 


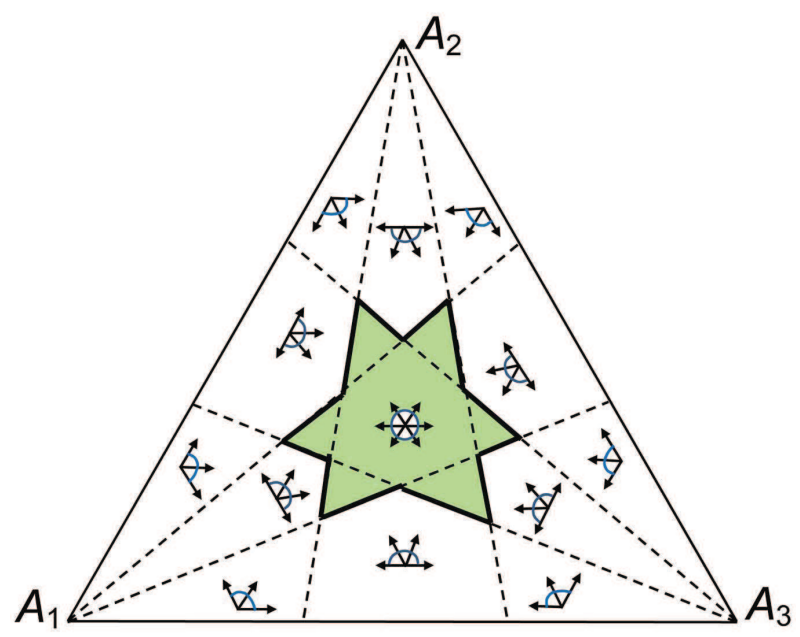

Figure 2. Partial equilibria of the reversible cycle with interval restrictions on the equilibrium constants. The lines of partial equilibria $A_{i} \rightleftharpoons A_{j}$, which correspond to the end of the intervals of equilibrium constants, split the triangle into several compartments. The borders of these compartments are combined from the intervals of the dashed lines. These dashed lines correspond to the minima and maxima of the equilibrium constants $\kappa_{j} / \kappa_{-j}$. In each compartment, the cone (the angle) of possible directions of $\dot{c}$ is given. This is a proper cone (an angle that is less than $\pi$ ) outside the equilibria strips, a halfplane in an equilibrium strip of a single reaction, and a whole plane in the intersection of two such strips. The area of the possible equilibria (the angle of possible directions of $\dot{c}$ is the whole plane) is outlined by bold line and colored in green.

\subsection{Transformation to fully reversible system}

Let us use the local equivalence of reversible and general Markov chains with the same equilibrium [6] (this is a particular case of local equivalence of systems with detailed and complex balance [7]) and represent the system (2.2) as a particular case of differential inclusion for a reversible reaction network $A_{1} \rightleftharpoons A_{2} \rightleftharpoons A_{3} \rightleftharpoons A_{1}, 2 A_{1} \rightleftharpoons 3 A_{2}$ (with possible extension of the interval of constants).

For every first order kinetics with given equilibrium the velocity vector $\dot{c}$ at each state $c$ may be expressed as a velocity vector for a first order system with detailed balance and the same equilibrium (the choice of this system with detailed balance depends on the state $c$ ). The cone of the possible velocity vectors is piecewise constant (Fig. 1).

The equilibrium concentrations $c_{i}^{*}$ in the irreversible cycle satisfy the following identities:

$$
k_{1} c_{1}^{*}=k_{2} c_{2}^{*}=k_{3} c_{3}^{*}, \quad \frac{c_{i}^{*}}{c_{j}^{*}}=\frac{k_{j}}{k_{i}}
$$

Instead of the irreversible cycle of linear reactions we will take the reversible cycle

$$
A_{1} \underset{\kappa-1}{\stackrel{\kappa_{1}}{\rightleftharpoons}} A_{2} \underset{\kappa_{-2}}{\stackrel{\kappa_{2}}{\rightleftharpoons}} A_{3} \underset{\kappa_{-3}}{\stackrel{\kappa_{3}}{\rightleftharpoons}} A_{1}
$$

with the interval restrictions on the equilibrium constants (the ratios of the reaction rate constants $\kappa_{j} / \kappa_{-j}$ )

$$
\frac{\min k_{2}}{\max k_{1}} \leq \frac{\kappa_{1}}{\kappa_{-1}} \leq \frac{\max k_{2}}{\min k_{1}}, \quad \frac{\min k_{3}}{\max k_{2}} \leq \frac{\kappa_{2}}{\kappa_{-2}} \leq \frac{\max k_{3}}{\min k_{2}}, \quad \frac{\min k_{1}}{\max k_{3}} \leq \frac{\kappa_{3}}{\kappa_{-3}} \leq \frac{\max k_{1}}{\min k_{3}}
$$

The detailed balance condition should also hold for the constants $\kappa_{ \pm j}$ :

$$
\kappa_{1} \kappa_{2} \kappa_{3}=\kappa_{-1} \kappa_{-2} \kappa_{-3}
$$




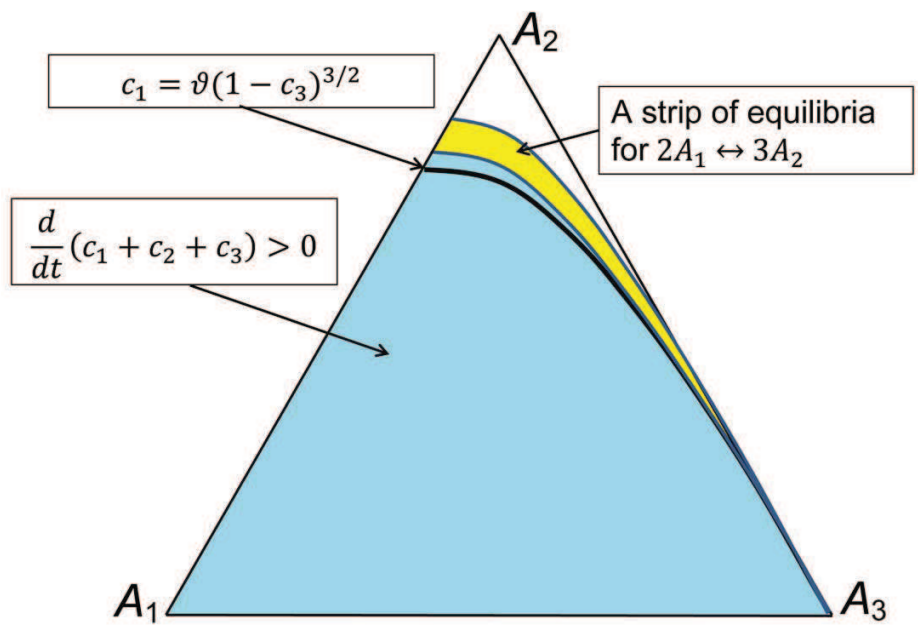

FIgURE 3 . The equilibrium strip of the reaction $2 A_{1} \rightleftharpoons 3 A_{2}$ (yellow) and the area where $\sum_{i} \dot{c}_{i}>0$ (blue) rescaled from the triangle with $\sum c_{i}=\varepsilon$ to the unit triangle

In the graphic illustrations below we use the symmetric intervals for $\kappa_{i} / \kappa_{-i}$ in the form

$$
\frac{1}{\omega} \leq \frac{\kappa_{1}}{\kappa_{-1}} \leq \omega
$$

for some $\omega>1$. Every interval could be included into such a symmetric one. Therefore, if we demonstrate persistence for the systems with symmetric intervals then it the persistence for all positive intervals is also proven.

The equilibria for this cycle satisfy the conditions

$$
\kappa_{1} c_{1}^{*}=\kappa_{-1} c_{2}^{*}, \kappa_{2} c_{2}^{*}=\kappa_{-2} c_{3}^{*}, \kappa_{3} c_{3}^{*}=\kappa_{-3} c_{1}^{*}
$$

These conditions provide the same range of equilibrium concentrations for the reversible and irreversible cycles. Therefore, the possible value of $\dot{c}$ for the irreversible cycle in the given interval of reaction rate constants always belongs to the cone of possible values of $\dot{c}$ of the reversible cycle under given restrictions.

For the reversible cycle the reaction rates are

$$
r_{1}=\kappa_{1} c_{1}-\kappa_{-1} c_{2}, r_{2}=\kappa_{2} c_{2}-\kappa_{-2} c_{3}, r_{3}=\kappa_{3} c_{3}-\kappa_{-3} c_{1}
$$

The differential inclusion for the linear cycle is represented in Fig. 2. There are three types of areas: (i) area where the equilibria may be located and the direction of $\dot{c}$ may coincide with any vector of the linear subspace $\sum_{i} \dot{c}_{i}=0$, (ii) areas where direction of one reaction is indefinite but the signs of two other reactions rates are fixed, and (iii) areas where signs of all reaction rates are fixed. The cones (angles) of possible vectors $\dot{c}$ are drown in Fig. 2.

\subsection{Separation of equilibria for different subsystems near origin}

For the linear system the scheme presented in Fig. 2 does not depend on the positive value of the balance $\sum_{i} c_{i}=\varepsilon$. We can just rescale $c_{i} \leftarrow c_{i} / \varepsilon$ and return to the unit triangle with the unit sum of $c_{i}$. The situation is different for the nonlinear reaction $2 A_{1} \rightleftharpoons 3 A_{2}$. Consider the "equilibrium strip" where the reaction rate $r_{4}=k_{4} c_{1}^{2}-k_{-4} c_{2}^{3}$ may be zero for the admissible reaction rate constants: 


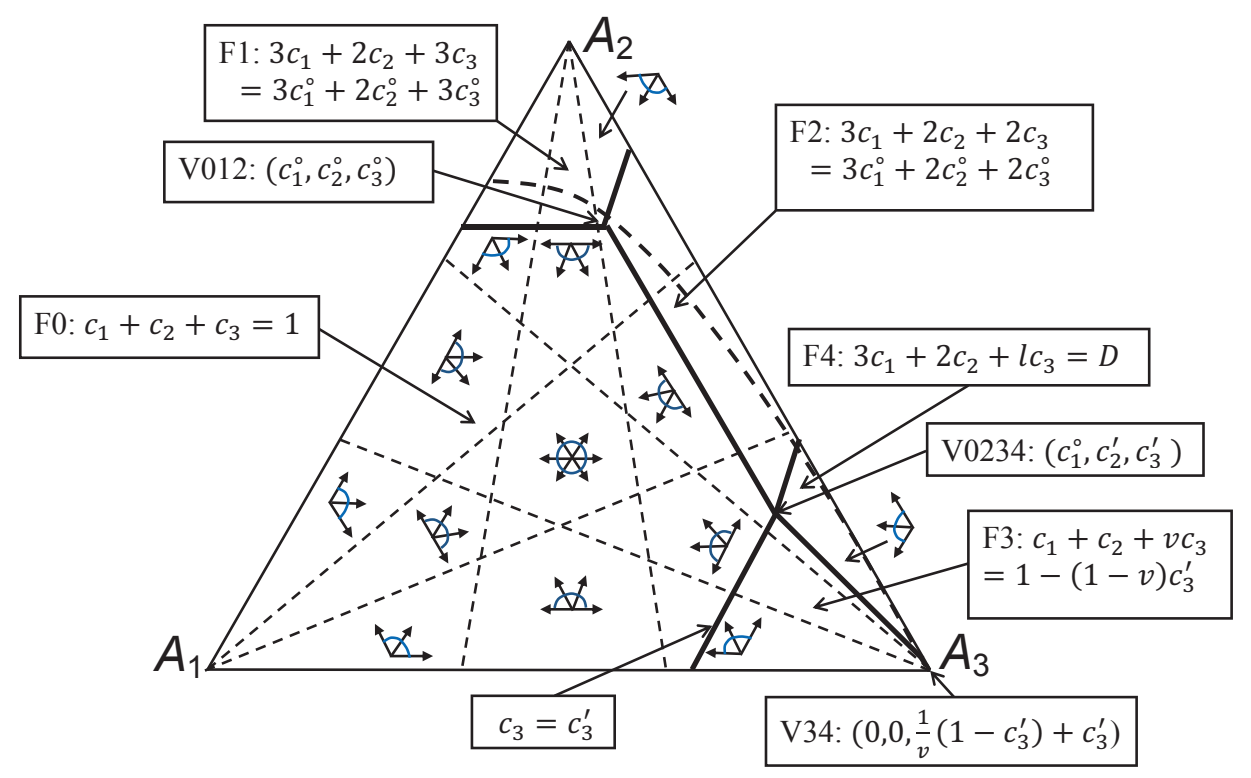

FIGURE 4. Faces of the peeled invariant set in the central projection onto unit triangle.

The borders between faces are highlighted by bold.

$$
\frac{\min k_{-4}}{\max k_{4}} \leq \frac{c_{1}^{2}}{c_{2}^{3}} \leq \frac{\max k_{-4}}{\min k_{4}}
$$

Let us take this strip on the plane $\sum_{i} c_{i}=\varepsilon$ and return it to the unit triangle by rescaling $\left(c_{i} \leftarrow c_{i} / \varepsilon\right)$. For small $\varepsilon$ this strip approaches the $\left[A_{2}, A_{3}\right]$ edge of the triangle. It is situated between the line

$$
c_{1}=\sqrt{\varepsilon} \sqrt{\frac{\max k_{-4}}{\min k_{4}}}\left(1-c_{3}\right)^{3 / 2}
$$

and the segment $\left[A_{2}, A_{3}\right]$. Further we use the notation $\vartheta$ for the coefficient in this formula:

$$
\vartheta=\sqrt{\varepsilon} \sqrt{\frac{\max k_{-4}}{\min k_{4}}}
$$

The line

$$
c_{1}=\vartheta\left(1-c_{3}\right)^{3 / 2}
$$

separates the equilibrium strip of the reaction $2 A_{1} \rightleftharpoons 3 A_{2}$ (where $r_{4}=0$ for some admissible combinations of the reaction rate constants) from the area where $r_{4}>0$ (i.e. $k_{4}\left(\varepsilon c_{1}\right)^{2}-k_{-4}\left(\varepsilon c_{2}\right)^{3}>0$ for all admissible $k_{4}, k_{-4}$. (We use the rescaling from the triangle with $\sum c_{i}=\varepsilon$ to the unit triangle without further comments.)

This line is tangent to the segment at the vertex $A_{3}$ (Fig. 3). On the other side of the line the time derivative of $\sum_{i} c_{i}$ is positive:

$$
\sum_{i} \dot{c}_{i}=r_{4}>0
$$




\subsection{Faces of peeled set and separation from origin}

Let us describe first the structure of the peeled set. Select for peeling the set $U=\left\{c \mid \sum_{i} c_{i} \geq \varepsilon, c_{i} \geq 0\right\}$. The structure of peeling scaled to $c_{1}+c_{2}+c_{3}=1$ is presented in Fig. 4 It appears that the piecewise linear peeling is sufficient. There are five faces different from the coordinate planes. The face F0 is a polygon on the plane $\sum c_{i}=1$. The face F1 is situated at the $A_{2}$ corner. It is produced by the peeling parallel to $\operatorname{Span}\left\{\gamma_{3}, \gamma_{4}\right\}$. The plane of $\mathrm{F} 1$ is given by the equation $3 c_{1}+2 c_{2}+3 c_{3}=$ const. The face $\mathrm{F} 2$ is presented by a parallelogram at the middle of the edge $\left[A_{2}, A_{3}\right]$ (Fig. 4). It covers the intersection of the equilibrium strips of the reactions $2 A_{1} \rightleftharpoons 3 A_{2}$ and the reaction $A_{2} \rightleftharpoons A_{3}$. F2 is produced by the peeling parallel to $\operatorname{Span}\left\{\gamma_{2}, \gamma_{4}\right\}$. The plane is given by the equation $3 c_{1}+2 c_{2}+2 c_{3}=$ const. Its intersection with the plane $c_{1}+c_{2}+c_{3}=1$ is a straight line $c_{1}=c_{1}^{\circ}, c_{2}+c_{3}=1-c_{1}^{\circ}$ for a sufficiently small $c_{1}^{\circ}>0$.

The final fragment of peeling is situated near the vertex $A_{3}$ (Fig. 4). It consists of two triangles. The first (F3) is a fragment of a plane $c_{1}+c_{2}+v c_{3}=$ const $(0<v<1)$. Parameter $v$ is defined from the condition of positive invariance below.

The second triangle (F4) situated near the vertex $A_{3}$ is parallel to $\gamma_{4}$ and the common edge with F3. The general plane parallel to $\gamma_{4}$ is given by the equation $3 c_{1}+2 c_{2}+l c_{3}=D$. We will define the parameters $l$ and $D$ from the vertices of the face F3,

Let us define the parameters of this peeling. At the $A_{2}$ corner the peeling is parallel to $\operatorname{Span}\left\{\gamma_{3}, \gamma_{4}\right\}$. The plane can be given by the equation $3 c_{1}+2 c_{2}+3 c_{3}=$ const. The edge between this face and the face $\sum c_{i}=1$ belongs to the straight line $c_{2}=c_{2}^{\circ}, c_{1}+c_{3}=1-c_{2}^{\circ}$. The level $c_{2}^{\circ}$ should be selected above all the equilibria of the linear reactions (Fig. 2) but below the intersection of the curve (2.4) with the right border of the equilibrium strip of the reaction $A_{1} \rightleftharpoons A_{3}$ given by the equation $c_{3}=c_{1} \max \left\{\frac{\kappa_{-3}}{\kappa_{3}}\right\}$. For the intersection we have

$$
c_{3}=\vartheta \max \left\{\frac{\kappa_{-3}}{\kappa_{3}}\right\}\left(1-c_{3}\right)^{3 / 2}
$$

Therefore, at this point

$$
c_{3}<\vartheta \max \left\{\frac{\kappa_{-3}}{\kappa_{3}}\right\}
$$

and $c_{1}<\vartheta$ on the line (2.4). Therefore, we can select

$$
c_{2}^{\circ}=1-\vartheta\left(\max \left\{\frac{\kappa_{-3}}{\kappa_{3}}\right\}+1\right)
$$

This $c_{2}^{\circ}$ is smaller than the value of $c_{2}$ at the intersection and for sufficiently small $\vartheta$, the line $c_{2}=c_{2}^{\circ}$ is close to the vertex $A_{2}$ and does not intersect the area of possible equilibria of linear reactions.

Consider intersection of the straight line $c_{2}=c_{2}^{\circ}, c_{1}+c_{3}=1-c_{2}^{\circ}$ with the curve (2.4) and evaluate the value of $c_{1}$ at this intersection from above: $c_{1}=\vartheta\left(c_{2}^{\circ}+c_{1}\right)^{3 / 2}, c_{1}<\vartheta$, hence, $c_{1}<c_{1}^{\circ}=\vartheta\left(c_{2}^{\circ}+\vartheta\right)^{3 / 2}$.

Thus, the vertex V012 at the intersection of three faces, F0, F1, and F2 is selected as $\left(c_{1}^{\circ}, c_{2}^{\circ}, c_{3}^{\circ}\right)$, where

$$
\begin{gathered}
c_{1}^{\circ}=\vartheta\left(1-\vartheta \max \left\{\frac{\kappa_{-3}}{\kappa_{3}}\right\}\right)^{3 / 2} \\
c_{2}^{\circ}=1-\vartheta\left(\max \left\{\frac{\kappa_{-3}}{\kappa_{3}}\right\}+1\right) \\
c_{3}^{\circ}=1-c_{1}^{\circ}-c_{2}^{\circ}=\vartheta\left(1+\max \left\{\frac{\kappa_{-3}}{\kappa_{3}}\right\}-\left(1-\vartheta \max \left\{\frac{\kappa_{-3}}{\kappa_{3}}\right\}\right)^{3 / 2}\right)
\end{gathered}
$$

To check that this point is outside the equilibrium strip of the reaction $A_{1} \rightleftharpoons A_{3}$, we calculate

$$
\frac{c_{3}^{\circ}}{c_{1}^{\circ}}=\frac{1+\max \left\{\frac{\kappa_{-3}}{\kappa_{3}}\right\}}{\left(1-\vartheta \max \left\{\frac{\kappa_{-3}}{\kappa_{3}}\right\}\right)^{3 / 2}}-1>\max \left\{\frac{\kappa_{-3}}{\kappa_{3}}\right\}
$$


The next group of parameters we have to identify are the coordinates of the vertex V0234 $\left(c_{1}^{\prime}, c_{2}^{\prime}, c_{3}^{\prime}\right)$ at the intersection of four faces F0, F2, F3, and F4. We will define it as the intersection of F0, F2, and F3 and then use its coordinated for definition of parameters of F4. One coordinate, $c_{1}^{\prime}$ is, obviously, $c_{1}^{\prime}=c_{1}^{\circ}$ because the intersection of $\mathrm{F} 2$ and $\mathrm{F} 0$ is parallel to $\gamma_{2}$, i.e. it is parallel to the edge $\left[A_{2}, A_{3}\right]$ of the unit triangle and $c_{1}$ is constant on this edge. Another coordinate, $c_{3}^{\prime}$ can be easily determined from the condition that the line $c_{3}=c_{3}^{\prime}$ in the unit triangle should not intersect the strips of equilibria for the reactions $A_{2} \rightleftharpoons A_{3}$ and $A_{1} \rightleftharpoons A_{3}$. Immediately, these condition give the inequalities that should hold for all admissible reaction rate constants:

$$
c_{3}^{\prime}>\frac{\kappa_{-3}}{\kappa_{3}+\kappa_{-3}}, \quad c_{3}^{\prime}>\frac{\kappa_{2}}{\kappa_{2}+\kappa_{-2}}
$$

Finally,

$$
c_{3}^{\prime}>\max \left\{\frac{1}{\min \left\{\frac{\kappa_{3}}{\kappa_{-3}}\right\}+1}, \frac{1}{1+\min \left\{\frac{\kappa_{-2}}{\kappa_{2}}\right\}}\right\}
$$

We can take $c_{3}^{\prime}$ between this maximum and 1 , for example we propose

$$
c_{3}^{\prime}=\frac{1}{2}+\frac{1}{2} \max \left\{\frac{1}{\min \left\{\frac{\kappa_{3}}{\kappa_{-3}}\right\}+1}, \frac{1}{1+\min \left\{\frac{\kappa_{-2}}{\kappa_{2}}\right\}}\right\}
$$

For sufficiently small $\vartheta$, the inequality $c_{3}^{\prime}+c_{1}^{\circ}<1$ holds, and we can take $c_{2}^{\prime}=1-c_{3}^{\prime}-c_{1}^{\circ}>0$.

If we know $c_{3}^{\prime}$ and $v$ then we know the equation of the plane $\mathrm{F} 4$ :

$$
c_{1}+c_{2}+v c_{3}=1-(1-v) c_{3}^{\prime}
$$

We also find immediately the coordinates of the vertex V34, the intersection of F3 (and F4) with the coordinate axis $A_{3}$. This vertex is $\left(0,0, \frac{1}{v}\left(1-c_{3}^{\prime}\right)+c_{3}^{\prime}\right)$.

Let us define the parameters $l$ and $D$ for the face F4. This face should include the vertices V0234 $\left(c_{1}^{\circ}, c_{2}^{\prime}, c_{3}^{\prime}\right)$ and $\operatorname{V} 34\left(0,0, \frac{1}{v}\left(1-c_{3}^{\prime}\right)+c_{3}^{\prime}\right)$. Therefore,

$$
l=v\left(2+\frac{c_{1}^{\circ}}{c_{1}^{\circ}+c_{2}^{\prime}}\right), D=3 c_{1}^{\circ}+2 c_{2}^{\prime}+l c_{3}^{\prime}
$$

To demonstrate the positive invariance of the peeled set we have to evaluate the sign of the inner product of $\dot{c}$ onto the inner normals to the faces on the faces.

The time derivatives of the concentrations are

$$
\dot{c}_{1}=-r_{1}+r_{3}-2 r_{4}, \dot{c}_{2}=r_{1}-r_{2}+3 r_{4}, \dot{c}_{3}=r_{2}-r_{3}
$$

The signs of some reaction rates are unambiguously defined on the faces:

- On F0 $r_{4}>0$

- On F1 $r_{1}<0$, and $r_{2}>0$;

- On F2 $r_{1}<0$, and $r_{3}>0$;

- On F3 $r_{2}<0, r_{3}>0$, and $r_{4}>0$;

- On F4 $r_{1}<0, r_{2}<0$, and $r_{3}>0$.

The inner products of $\dot{c}$ onto the inner normals to the faces are:

- On F0 $\frac{d}{d t}\left(c_{1}+c_{2}+c_{3}\right)=r_{4}>0$;

- On F1 $\frac{d}{d t}\left(3 c_{1}+2 c_{2}+3 c_{3}\right)=-r_{1}+r_{2}>0$;

- On F2 $\frac{d}{d t}\left(3 c_{1}+2 c_{2}+2 c_{3}\right)=-r_{1}+r_{3}>0$; 
- On F3 $\frac{d}{d t}\left(c_{1}+c_{2}+v c_{3}\right)=(1-v)\left(-r_{2}+r_{3}\right)+r_{4}>0(0<v<1)$

- On F4 $\frac{d}{d t}\left(3 c_{1}+2 c_{2}+l c_{3}\right)=-r_{1}-(2-l) r_{2}+(3-l) r_{3}<0$ if $0<l<2$.

Thus, the peeled set is positively invariant if $0<l<2$. This means

$$
0<v<\frac{1}{1+\frac{c_{1}^{\circ}}{2\left(c_{1}^{\circ}+c_{2}^{\prime}\right)}}
$$

It is sufficient to take $0<v<\frac{2}{3}$ because of obvious inequality.

We have demonstrated that for any given range of positive kinetic constants any solution of the kinetic inclusion for the system (1.1) cannot approach the origin when $t \rightarrow \infty$. We have constructed a piecewiselinear surface that isolated the $\varepsilon$-vicinity of the origin from the outside. If $\varepsilon>0$ is sufficiently small then this surface cannot be intersected by the solutions of the kinetic inclusion in the motion from the outside to the origin.

\section{Discussion}

The forward-invariant peeling is a universal procedure for producing forward-invariant sets for kinetic equations and inclusions. The peeling procedure used in this toy-example for isolation from zero differs from the general greedy peeling [7]. (It is a simplified version of the greedy peeling.) We have guessed the structure of the corner near $A_{3}$ and build two plain faces, F3 and F4, instead of a sequence of the curvilinear "cylindric" faces. This piecewise peeling is not minimal but is simpler for drawing.

Acknowledgements. I am very grateful to Anne Shiu for encouraging comments.

\section{References}

[1] G. Craciun. Toric differential inclusions and a proof of the global attractor conjecture. 2015. arXiv:1501.02860 [math.DS]

[2] G. Craciun, F. Nazarov, C. Pantea. Persistence and permanence of mass-action and power-law dynamical systems. SIAM J. Appl. Math. 73 (2013), 305-329.

[3] A.N. Gorban. Invariant sets for kinetic equations. React. Kinet. Catal. Lett., 10 (1979), 187-190.

[4] A.N. Gorban. On the problem of boundary equilibria. React. Kinet. Catal. Lett., 15 (1980), 315-319. (In Russian)

[5] A.N. Gorban. Equilibrium encircling. Equations of Chemical Kinetics and Their Thermodynamic Analysis. Nauka, Novosibirsk, 1984. (In Russian)

[6] A.N. Gorban. Local equivalence of reversible and general Markov kinetics. Physica A, 392 (2013), $1111-1121$. arXiv:1205.2052 [physics.chem-ph].

[7] A.N. Gorban. General H-theorem and entropies that violate the second law. Extended postprint, 2014. arXiv:1212.6767 [cond-mat.stat-mech].

[8] M. Gopalkrishnan, E. Miller, A. Shiu. A geometric approach to the Global Attractor Conjecture, SIAM Journal on Applied Dynamical Systems 13 (2014), 758-797. arXiv:1305.5303 [math.DS]

[9] A.I. Vol'pert. Qualitative methods of investigation of equations of chemical kinetics, Institute of Chemical Physics, Chernogolovka, Preprint, 1976. (In Russian)

[10] G.S. Yablonskii, V.I. Bykov, A.N. Gorban, V.I. Elokhin. Kinetic Models of Catalytic Reactions. Elsevier, Amsterdam, The Netherlands, 1991. 